\title{
Baastrup's Disease Is Associated with Recurrent of Sciatica after Posterior Lumbar Spinal Decompressions Utilizing Floating Spinous Process Procedures
}

\author{
Masao Koda ${ }^{1,2}$, Chikato Mannoji ${ }^{2}$, Masazumi Murakami ${ }^{2}$, Tomoaki Kinoshita ${ }^{3}$, \\ Jiro Hirayama ${ }^{4}$, Tomohiro Miyashita ${ }^{5}$ Yawara Eguchi ${ }^{5}$, Masashi Yamazaki ${ }^{6}$, Takane Suzuki ${ }^{7}$, \\ Masaaki Aramomi ${ }^{1}$, Mitsutoshi Ota ${ }^{1}$, Satoshi Maki ${ }^{1}$, Kazuhisa Takahashi ${ }^{1}$, Takeo Furuya ${ }^{1}$ \\ ${ }^{I}$ Department of Orthopedic Surgery, Chiba University Graduate School of Medicine, Chiba, Japan \\ ${ }^{2}$ Department of Orthopedic Surgery, Chiba Aoba Municipal Hospital, Chiba, Japan \\ ${ }^{3}$ Department of Orthopedic Surgery, Narashino Dai-ichi Hospital, Narashino, Japan \\ ${ }^{4}$ Department of Orthopedic Surgery, Chiba Medical Center, Chiba, Japan \\ ${ }^{5}$ Department of Orthopedic Surgery, Matsudo City Hospital, Matsudo, Japan \\ ${ }^{6}$ Department of Orthopedic Surgery, University of Tsukuba, Tsukuba, Japan \\ ${ }^{7}$ Department of Bioenvironmental Medicine, Chiba University Graduate School of Medicine, Chiba, Japan
}

Study Design: Retrospective case-control study.

Purpose: To determine whether kissing spine is a risk factor for recurrence of sciatica after lumbar posterior decompression using a spinous process floating approach.

Overview of Literature: Kissing spine is defined by apposition and sclerotic change of the facing spinous processes as shown in X-ray images, and is often accompanied by marked disc degeneration and decrement of disc height. If kissing spine significantly contributes to weight bearing and the stability of the lumbar spine, trauma to the spinous process might induce a breakdown of lumbar spine stability after posterior decompression surgery in cases of kissing spine.

Methods: The present study included 161 patients who had undergone posterior decompression surgery for lumbar canal stenosis using a spinous process floating approaches. We defined recurrence of sciatica as that resolved after initial surgery and then recurred. Kissing spine was defined as sclerotic change and the apposition of the spinous process in a plain radiogram. Preoperative foraminal stenosis was determined by the decrease of perineural fat intensity detected by parasagittal T1-weighted magnetic resonance imaging. Preoperative percentage slip, segmental range of motion, and segmental scoliosis were analyzed in preoperative radiographs. Univariate analysis followed by stepwise logistic regression analysis determined factors independently associated with recurrence of sciatica.

Results: Stepwise logistic regression revealed kissing spine $(p=0.024$; odds ratio, 3.80) and foraminal stenosis $(p<0.01$; odds ratio, 17.89) as independent risk factors for the recurrence of sciatica after posterior lumbar spinal decompression with spinous process floating procedures for lumbar spinal canal stenosis.

\footnotetext{
Received Feb 12, 2016; Revised Apr 20, 2016; Accepted May 6, 2016

Corresponding author: Masao Koda

Department of Orthopedic Surgery, Chiba University Graduate School of Medicine

1-8-1 Inohana, Chuo-Ku, Chiba 2608670, Japan

Tel: +81-43-226-2961, Fax: +81-43-224-5124, E-mail:masaokod@gmail.com
} 
Conclusions: When a patient shows kissing spine and concomitant subclinical foraminal stenosis at the affected level, we should sufficiently discuss the selection of an appropriate surgical procedure.

Keywords: Lumbar stenosis; Postoperative complications; Sciatica

\section{Introduction}

Kissing spine, first described by Baastrup [1], is defined by apposition and sclerotic change of the facing spinous processes as shown in X-ray images. It is often accompanied with marked disc degeneration and decrement of disc height. Those changes suggest the dispersion of weight bearing to the spinous process associated with the progression of spondylosis [2]. However, the clinical significance of kissing spine is not fully understood. In particular, the influence of kissing spine on the structural strength of the lumbar spine is unclear.

If kissing spine significantly contributes to weight bearing and the stability of the lumbar spine, trauma to the spinous process might induce a breakdown of lumbar spine stability after posterior decompression surgery in cases of kissing spine. Itoi et al. [3] reported that several patients with kissing spine showed recurrence of sciatica, which was attributed to foraminal stenosis after posterior lumbar spine decompression surgery using a spinous process splitting procedure, which results in a floating spinous process.

The purpose of the present study was to determine whether kissing spine is a risk factor for recurrence of sciatica after lumbar posterior decompression using a spinous process splitting procedure.

\section{Materials and Methods}

The retrospective case-controlled study included patients who had undergone posterior decompression surgery using a spinous process floating approach [4-6] to lumbar canal stenosis at our institutions from January 2009 to December 2011 and were followed-up at least 1 year. We analyzed cases in 161 patients, including 129 men and 32 women. The average age of the patients at surgery was 71.2 years (range, 51-86 years) and the average follow-up period was 15.6 months (range, 12-48 months).

Two surgical procedures were included in the present series: the spinous process splitting approach $[5,6]$ and a spinous process retracting approach [4]. Both produced subsequent floating of the spinous process. Briefly, the spinous process splitting approach was performed with preservation of paraspinal muscle on both sides. The spinous process was cut in the middle and split to both sides with preservation of its muscle attachment. Then the split spinous process-paraspinal muscle complex was retracted to either side to obtain a clear operative field. After decompression, split parts of the spinous process were sutured to each other to obtain apposition $[5,6]$.

The other posterior approach was a spinous process retracting method. It was performed with preservation of supraspinous and interspinous ligaments and with preservation of the paraspinal muscle on one side. Next, the affected spinous process was cut at its base using an osteotome and the preserved spinous process-paraspinal muscle complex was retracted to obtain an operative field. After decompression, the spinous process returned to its original position with suturing to the lumbosacral fascia [4].

We defined the recurrence of sciatica as that which resolved after initial surgery, but then recurred, in which pain intensity could not be attenuated by analgesics alone (needed radicular infiltration, epidural infiltration, or revision surgery, etc). The average duration of pain free after the initial surgery was 6.5 months (range, 4-12 months). Thus, we excluded residual sciatica caused, for example, by insufficient decompression. We also excluded juxtafacet cysts revealed by magnetic resonance imaging (MRI). Kissing spine was defined by plain radiographic image findings, which included sclerotic change of the spinous process and apposition of the spinous process at the affected level during extension [2]. Preoperative foraminal stenosis was determined as described [7] by the decrease of perineural fat intensity detected by parasagittal T1weighted MRI at the affected level of the lumbar spine. The severity of foraminal stenosis was graded 0 if the foramina were without pathology (normal dorsolateral border of the intervertebral disk and normal form at the 
foraminal epidural fat); grade 1 if slight foraminal stenosis and deformity of the epidural fat were seen, with the remaining fat still completely surrounding the exiting nerve root; grade 2 if marked foraminal stenosis with epidural fat only partially surrounding the nerve root was seen; and grade 3 for advanced stenosis with obliteration of the epidural fat [7]. Grade 3 was considered as foraminal stenosis. We analyzed preoperative percentage slip, segmental range of motion, and segmental scoliosis in preoperative radiographs. First, we performed multiple co-linearity analyses. Next, the impact of the abovementioned factors on recurrence of sciatica was analyzed using univariate analysis. The factors showing $p<0.1$ were subjected to stepwise logistic regression analysis to determine which independent factors had an impact on the recurrence of sciatica. JMP software (ver. 10; SAS Institute Japan, Tokyo, Japan) was used for statistical analyses.

\section{Results}

The patient background data are shown in Table 1. Kissing spine was observed in 45 patients. There were significant difference in age and male:female ratio between the patients with or without kissing spine (average age was 74.3 in the patients with kissing spine and 70.0 in the patients without kissing spine; male:female ratio was $8.6: 1$ in the patients with kissing spine and $1.25: 1$ in the patients without kissing spine).

First, we analyzed possible risk factors for recurrence of sciatica with multiple co-linearity analyses. The analyzed factors were the existence of kissing spine, age, sex, and preoperative radiographic findings including foraminal stenosis, segmental scoliosis, \% slip, and segmental range of motion. There was co-linearity between kissing spine and age $(p=0.01)$, kissing spine and sex $(p<0.001)$, local scoliosis and segmental range of motion $(p=0.0001)$. Univariate analysis of possible risk factors indicated that kissing spine ( $p=0.0002)$, sex ( $p=0.0032)$, segmental range of motion $(p=0.031)$, and foraminal stenosis $(p<0.0001)$ were significant, whereas age $(p=0.106)$, segmental scoliosis $(p=0.26)$, and $\%$ slip $(p=0.21)$ were not, and therefore were excluded from further analysis.

The factors selected by univariate analysis were then analyzed by stepwise logistic regression, which revealed that kissing spine $(p=0.01)$ and foraminal stenosis $(p=0.04)$ were independent risk factors for the recurrence of sciatica after posterior lumbar spinal decompression with
Table 1. Patient background data

\begin{tabular}{lc} 
Factors & Total $(\mathrm{n}=161)$ \\
\hline Age $(\mathrm{yr})$ & $71.1(51-86)$ \\
Sex (male:female) & $128: 33$ \\
\hline Follow-up (mo) & $15.6(12-48)$ \\
\hline Preoperative radiographs & $1.2(0-20)$ \\
\hline Slip (\%) & $5.8(1-11)$ \\
\hline Segmental range of motion $\left(^{\circ}\right)$ & $3.0(0-9)$ \\
\hline Segmantal scoliosis $\left(^{\circ}\right)$ & $45 / 161(28.0)$ \\
\hline Kissing spine $(+)$ & $18 / 161(11.2)$ \\
\hline Foraminal stenosis $(+)$ &
\end{tabular}

Values are presented as median (range) or number (\%).

Table 2. Statistical analyses of risk factors for recurrent of sciatica after posterior lumbar spinal decompression with floating spinous process procedures

\begin{tabular}{ll} 
Possible risk factors & $p$-value \\
Univariate analysis & \\
\hline Age & 0.106 \\
\hline Sex & $0.0032^{* *}$ \\
\hline Kissing spine & $0.0002^{* *}$ \\
\hline Foraminal stenosis & $<0.0001^{* *}$ \\
\hline Segmental scoliosis & 0.26 \\
\hline \% Slip & 0.18 \\
\hline Segmental range of motion & $0.031^{*}$ \\
\hline Stepwise logistic regression & \\
\hline Sex & $0.78^{* *}$ \\
\hline Kissing spine & $0.01^{* *}$ \\
\hline Foraminal stenosis & $0.04^{*}$ \\
\hline Segmental range of motion & 0.06 \\
\hline
\end{tabular}

${ }^{*} p<0.05,{ }^{* *} p<0.01$.

spinous process floating procedures for lumbar spinal canal stenosis (Table 2). As for revision surgery, kissing spine patients tended to show higher incidence of revision surgery ( $8.9 \%$ in kissing patients and $2.6 \%$ in nonkissing spine patients, $p=0.08$ ).

\section{Representative case report}

A 68-year-old man suffered from bilateral posterior thigh pain after 10 minutes of walking. Preoperative X-ray imaging revealed apposition of the spinous processes at 
L4-5 during extension and sclerotic change of the inferior border of L4 and superior border of L5 spinous processes (Fig. 1A). Preoperative MRI showed moderate stenosis at the L4-5 level (Fig. 1B, C). The patient underwent posterior lumbar spine decompression using spinous processsplitting procedure (Fig. 1D). After surgery, bilateral posterior thigh pain disappeared and the patient was able to walk without pain for up to 1 hour. Three months after the surgery, the patient complained of left lower leg pain after several minutes of walking. MRI revealed sufficient central decompression at L4-5 (Fig. 1E); however, foraminal stenosis on the left side at $\mathrm{L} 4-5$ was detected on a paramedian sagittal MRI (Fig. 1F). Lower leg pain was re- lieved by left L4 radicular block with local analgesics (Fig. $1 G)$. During follow-up for three years after the recurrence of sciatica, the patient showed no apparent sciatica.

\section{Discussion}

The present study revealed that kissing spine and preoperative foraminal stenosis are independent risk factors for the recurrence of sciatica after posterior lumbar decompression with floating spinous process procedures.

Kissing spine is almost concomitant with disk degeneration and decrease of disk height, suggesting its contribution to posteriorly-shifted weight bearing of the lumbar
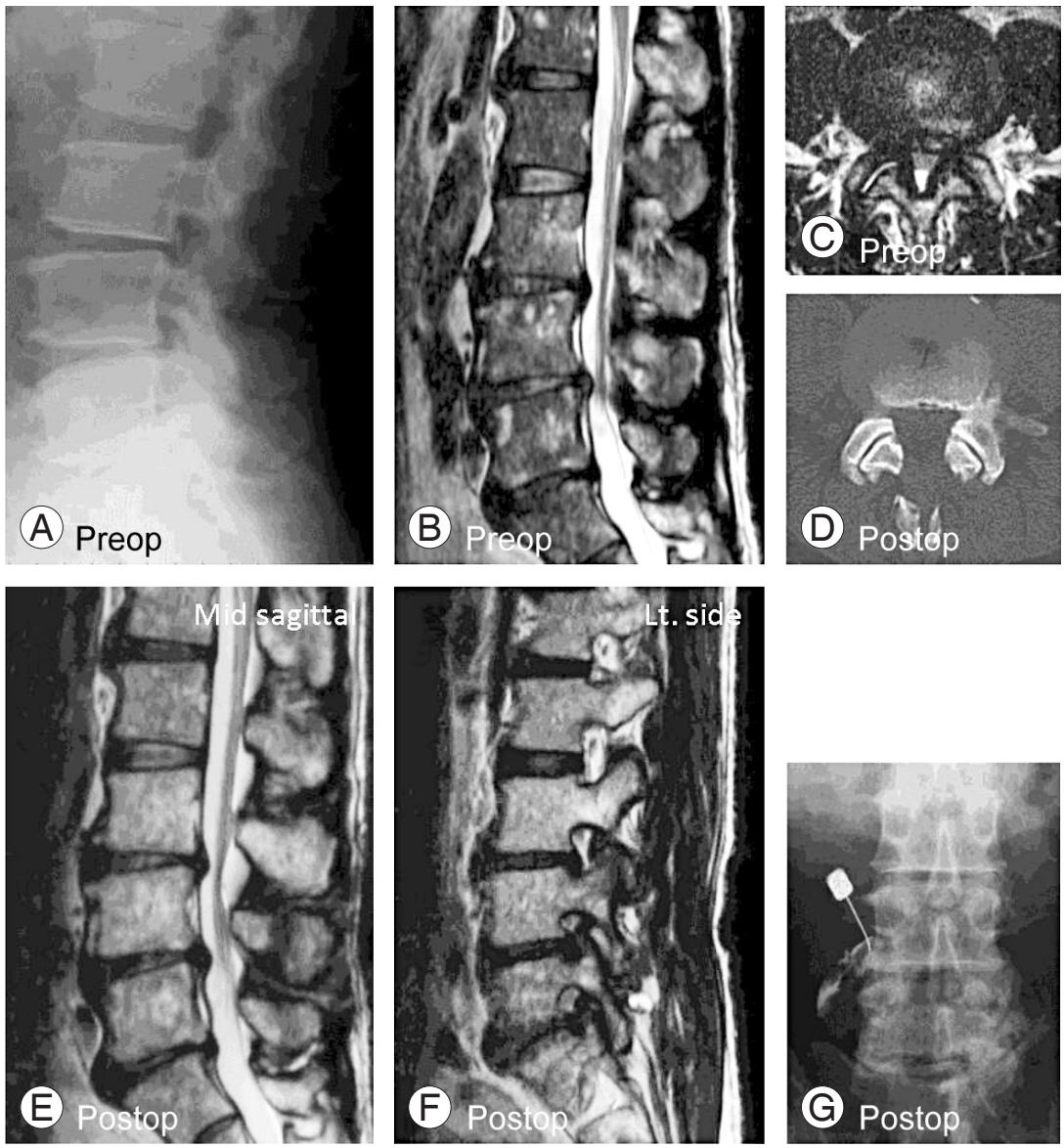

Fig. 1. Representative case. A 68-year-old man suffered from bilateral posterior thigh pain after 10 minutes of walking. Preoperative $X$-ray imaged kissing spine at $L 4 / 5$ level (A). Preoperative magnetic resonance imaging (MRI) showed moderate stenosis at the $L 4-5$ level $(\mathbf{B}, \mathbf{C})$. Following posterior lumbar spine decompression using spinous process-splitting procedure (D), bilateral posterior thigh pain disappeared and the patient was able to walk without pain for up to 1 hour. Three months after the surgery, the patient complained of left lower leg pain after several minutes of walking. MRI revealed sufficient central decompression at $L 4-5$ (E); however, foraminal stenosis on the left side at $L 4-5$ was detected on a paramedian sagittal MR image (F). Lower leg pain was relieved by left L4 radicular block with local analgesics (G). Preop, preoperative; Postop, postoperative. 
spine [8]. If the posteriorly-shifted weight bearing pattern is disrupted by posterior lumbar decompression using floating spinous process procedures, then sciatica as a result of foraminal stenosis that was subclinical before surgery might be manifested.

Previous reports describe possible risk factors for poor clinical outcome after lumbar decompression surgery as follows: severe constriction of the dural tube, preoperative periods [9], multiple segments involved, larger sagittal rotation $[10,11]$, and residual redundant nerve roots [12]. Itoi et al. [3] first described a case-series in which several patients with kissing spine showed postoperative recurrence of sciatica. They speculated that disruption of posteriorly-shifted weight bearing by invasion from kissing spine might result in the manifestation of subclinical foraminal stenosis [3]. The present result was consistent. The present results are the first to show that kissing spine might be the possible risk factor to the recurrence of sciatica after posterior lumbar spine decompression using a floating spinous process procedure.

As yet there is no consensus as to whether lumbar posterior decompression using floating spinous process procedures is suitable for patients with kissing spine. To clarify this point, further exploration (for example, of direct comparison between spinous process floating procedures $[4-6]$ versus spinous process intact procedures $[11,13,14]$ ) is needed. This is one of major limitations of the present study. Another limitation is underlying mechanism. Patients treated by conventional partial resection of spinous process and decompressive laminotomy should represent same problem if kissing spine really causes recurrent sciatica. We cannot draw any conclusion concerning whether recurrent sciatica is a specific problem with spinous process-floating posterior decompression procedures or there is significant biomechanical difference between spinous process-floating procedures and conventional laminotomy (or laminectomy). Further investigations including data analyses about relationship between conventional laminotomy and kissing spine are needed.

Although we cannot darw definitive conclusion, we recommend informing patients with kissing spine that floating spinous process procedures might induce a higher rate of sciatica recurrence. When a patient shows kissing spine and concomitant subclinical foraminal stenosis at the affected level, we should sufficiently discuss the selection of an appropriate surgical procedure.

\section{Conclusions}

In conclusion, kissing spine is one of the possible risk factors for recurrence of sciatica after posterior lumbar spinal decompression using floating spinous process procedures for decompression surgery for lumbar spinal canal stenosis. When a patient shows kissing spine and concomitant subclinical foraminal stenosis at the affected level, we should sufficiently discuss the selection of an appropriate surgical procedure.

\section{Conflict of Interest}

No potential conflict of interest relevant to this article was reported.

\section{References}

1. Baastrup CL. Proc. Spin. Vert. lumb und einige zwischen diesen liegenden gelenkbildungen mit pathologischen prozessen in dieser region. Rofo 1933; 48:430-5.

2. Clifford PD. Baastrup disease. Am J Orthop (Belle Mead NJ) 2007;36:560-1.

3. Itoi A, Shimamura Y, Muta T, Kaneko K. Problems of the posterior lumbar approach technique that does not keep kissing spines. J Spine Res 2010;1:1449-56.

4. Kinoshita T, Ohki I, Roth KR, Amano K, Moriya H. Results of degenerative spondylolisthesis treated with posterior decompression alone via a new surgical approach. J Neurosurg 2001;95:11-6.

5. Watanabe K, Hosoya T, Shiraishi T, Matsumoto M, Chiba K, Toyama Y. Lumbar spinous process-splitting laminectomy for lumbar canal stenosis: technical note. J Neurosurg Spine 2005;3:405-8.

6. Watanabe K, Matsumoto M, Ikegami T, et al. Reduced postoperative wound pain after lumbar spinous process-splitting laminectomy for lumbar canal stenosis: a randomized controlled study. J Neurosurg Spine 2011;14:51-8.

7. Wildermuth S, Zanetti M, Duewell S, et al. Lumbar spine: quantitative and qualitative assessment of positional (upright flexion and extension) MR imaging and myelography. Radiology 1998;207:391-8.

8. Maes R, Morrison WB, Parker L, Schweitzer ME, Carrino JA. Lumbar interspinous bursitis (Baastrup disease) in a symptomatic population: prevalence on 
magnetic resonance imaging. Spine (Phila Pa 1976) 2008;33:E211-5.

9. Jonsson B, Annertz M, Sjoberg C, Stromqvist B. A prospective and consecutive study of surgically treated lumbar spinal stenosis. Part II: Five-year followup by an independent observer. Spine (Phila Pa 1976) 1997;22:2938-44.

10. Iguchi T, Kurihara A, Nakayama J, Sato K, Kurosaka M, Yamasaki K. Minimum 10-year outcome of decompressive laminectomy for degenerative lumbar spinal stenosis. Spine (Phila Pa 1976) 2000;25:1754-9.

11. Matsumura A, Namikawa T, Terai H, et al. The influence of approach side on facet preservation in microscopic bilateral decompression via a unilateral approach for degenerative lumbar scoliosis. Clinical article. J Neurosurg Spine 2010;13:758-65.
12. Yokoyama K, Kawanishi M, Yamada M, et al. Clinical significance of postoperative changes in redundant nerve roots after decompressive laminectomy for lumbar spinal canal stenosis. World Neurosurg 2014; 82:e825-30.

13. Musluman AM, Cansever T, Yilmaz A, Cavusoglu H, Yuce I, Aydin Y. Midterm outcome after a microsurgical unilateral approach for bilateral decompression of lumbar degenerative spondylolisthesis. J Neurosurg Spine 2012;16:68-76.

14. Tanaka N, Nakanishi K, Kamei N, et al. Clinical results of microsurgical bilateral decompression via unilateral approach for lumbar canal stenosis with multiple-level involvement. Eur J Orthop Surg Traumatol 2015;25 Suppl 1:S191-8. 